\title{
Implementación del Algoritmo Evolutivo Multi-Objetivo de Frente de Pareto (SPEA) para la Planeación de Sistemas Eléctricos de Distribución incluyendo Huecos de Voltaje
}

\author{
Carlos A. García ${ }^{(1)}$, Edwin García(2) y Fernando Villada ${ }^{(3)}$ \\ (1) Empresas Públicas de Medellín. Carrera 58 \# 52-125. Medellín, Antioquia, Colombia. \\ (e-mail: carlos.garcia.montoya@epm.com.co) \\ (2) Grupo de Investigación TESLA, Universidad de Antioquia. Calle 67 \# 53-108, 20-113. \\ Medellín, Antioquia, Colombia. (e-mail: edwin.garciaq@udea.edu.co) \\ (3) Departamento de Ingeniería Eléctrica, Universidad de Antioquia. Calle 67 \# 53-108, 20-421. \\ Medellín, Antioquia, Colombia (e-mail: fernando.villada@udea.edu.co)
}

Recibido Feb. 13, 2015; Aceptado Abr. 20, 2015; Versión final May. 23, 2015, Publicado Oct. 2015

\begin{abstract}
Resumen
En este trabajo se presenta la implementación del algoritmo evolutivo multi-objetivo de frente de Pareto (SPEA) para la planeación de la expansión de sistemas de distribución, considerando como funciones objetivos los costos de la inversión y el número de huecos de voltaje esperado por año. El algoritmo se implementa aplicándolo a un sistema de distribución real teniendo en cuenta algunos parámetros fijos del sistema, tales como el número de tramos, el número de nodos y la población inicial. También se consideran otras variables que son propias de los algoritmos evolutivos como el porcentaje en poblaciones distintas que se cruzan entre sí en una generación (cruce) y la mutación. Se concluye que la implementación de SPEA para la planeación de sistemas de distribución reales es una buena y eficiente herramienta computacional cuando se requiere considerar varias funciones objetivo.
\end{abstract}

Palabras clave: planeación de sistemas de distribución, calidad de la potencia, algoritmos evolutivos de frente de Pareto, SPEA, optimización multi-objetivo

\section{Implementing the Strength Pareto Evolutionary Algorithm (SPEA) for the Planning of Electrical Distribution Systems including Sag Voltage}

\begin{abstract}
This article describes the implementation of the multi-objective evolutionary algorithm Pareto front (SPEA) for the planning of distribution system expansion, taking as target functions the investment costs and the number of Sag voltages expected per year. The algorithm is implemented by applying it to real distribution system, given some fixed system parameters such as the number of network segments, number of nodes and the number of initial population. Other variables that are proper of evolutionary algorithms such as percentage of generation population that crosses another (cross) and mutation are taken into consideration. It is concluded that the implementation of SPEA for planning real distribution systems is a good and efficient computational tool when it is necessary to consider several objective functions.
\end{abstract}

Keywords: distribution system planning, power quality, evolutionary algorithm of Pareto front, SPEA, multi-objective optimization 


\section{INTRODUCCIÓN}

En las últimas décadas el sector eléctrico a nivel mundial ha sufrido grandes cambios en materia regulatoria, siempre con miras a mejorar la calidad del servicio y la potencia suministrada al usuario final. Para esto se han elaborado leyes que rigen el mercado de energía y el servicio, definiendo los límites permisibles para las variables y características del servicio. Con el objetivo de cumplir con las restricciones regulatorias y hacer uso eficiente de los recursos disponibles, las empresas de distribución han dedicado esfuerzos y recursos en planear la forma como el sistema debe estar preparado para atender contingencias operativas y disponer de capacidad suficiente para atender el crecimiento de la demanda, todo esto bajo condiciones de costoeficiencia y características técnicas básicas. Resolver lo anterior genera grandes demandas de tiempo y capacidades de cómputo dadas las dimensiones y variables que posee el sistema de distribución; además, la planeación del sistema no es una tarea que se pueda realizar por simple inspección, porque pueden existir millones de posibilidades factibles, lo cual ha llevado a emplear técnicas de optimización en la planeación de la distribución, y entre ellas unas de las más relevantes es el algoritmo evolutivo multi-objetivo SPEA, por su flexibilidad y alta selectividad, lo cual minimiza el problema y reduce los cálculos, así como el tiempo y la capacidad de cómputo requerida.

Son diferentes los objetivos que pueden trazarse en la planeación de la distribución. Todos estos dependen del entorno, del crecimiento de la demanda esperado, los recursos disponibles, las características del sistema, entre otros. Unos de los objetivos más comunes dentro de la planeación son: la minimización de los índices de calidad del servicio, minimización de la magnitud de la energía no suministrada, minimización de las inversiones realizadas en el sistema, entre otros.

Para optimizar estos objetivos muchas técnicas se han utilizado. En la última década se han popularizado las técnicas heurísticas, tal como se presenta en Lavorato et al. (2010), donde se emplea una heurística constructiva para resolver el problema de optimización del costo de inversión y el costo de las pérdidas operacionales. Otros trabajos se han enfocado en el diseño óptimo de la red bajo condiciones específicas (Carrano et al., 2006; Mendoza et al., 2006; Díaz-Dorado et al., 2003), donde se han empleado técnicas evolutivas para la definición de un diseño óptimo o un conductor óptimo para un sistema en especial.

Considerando que la reglamentación es cada vez más exigente con las empresas de distribución, se ha visto la necesidad de emplear técnicas de optimización para minimizar las inversiones realizadas en las redes considerando aspectos como confiabilidad, calidad del servicio y calidad de la energía. Para ello se han empleado técnicas como particle swarm, como se presenta en Ganguly et al. (2009), y algoritmos evolutivos, como se describen en (Pilo et al., 2007; Loreto y Contreras, 2011; Cossi et al., 2012).

Algunos trabajos plantean la optimización de la expansión del sistema de distribución considerando fundamentalmente aspectos económicos y constructivos, tal como se aprecia en García et al. (2012), pero solo considerando una función objetivo. En el presente trabajo se plantea un algoritmo evolutivo para la definición en el corto plazo de la planeación de un sistema de distribución, considerando en la optimización de dicha planeación dos funciones objetivos: la función de costos de la inversión en la expansión y el número de SAGS esperados por año sobre el sistema analizado. La función de costos a su vez está conformada por dos funciones: una de costos fijos y otra de costos variables, y ambas deben ser minimizadas. Para ello se emplea un algoritmo evolutivo multi objetivo, que para este caso es un SPEA (Strength Pareto Evolutionary Algorithm) Zitzler y Thiele (1999). A diferencia del trabajo citado en García et al. (2012), que solo evalúa una función objetivo, el presente trabajo dado su carácter multi-objetivo evalúa dos funciones, permitiendo la optimización de los SAGS esperados y el costo de la expansión del sistema, tal como se explica en la sección caso de estudio y resultados. El algoritmo evolutivo multi-objetivo SPEA presenta enormes ventajas aplicadas a optimizar problemas que involucran varias funciones objetivos. No obstante, existe una versión posterior llamada SPEA-2, la cual mejora aspectos como la agrupación de individuos con características similares, incremento de la densidad de soluciones pegadas a la curva del frente de Pareto, García (2010); pero en la literatura no hay aplicaciones de ella (así como tampoco de SPEA) al caso particular de la optimización de la planeación de los sistemas de distribución de energía eléctrica, por lo que es viable implementar en esta instancia el algoritmo SPEA para explorar su potencial en esta aplicación. Adicionalmente, existen otros algoritmos multi-objetivos como el NSGA y NSGA-II, que tienen sus ventajas frente al SPEA en la alta selectividad, lo cual los hace más relevantes en aplicaciones de tipo biológico García (2010). Por tanto, el algoritmo SPEA es una buena herramienta para la aplicación planteada en el presente trabajo.

Para lograr lo anterior en la sección optimización multi objetivo mediante algoritmos evolutivos se presentan los fundamentos de los algoritmos evolutivos, haciendo énfasis en el algoritmo implementado (SPEA), en la sección calidad de la energía en la planeación de la distribución se presenta el cálculo de los SAGS para la planeación del sistema de distribución del caso estudio. Luego, la sección formulación del modelo de planeación presenta el algoritmo implementado para esta tarea, considerando la calidad de la energía. La 
sección algoritmo implementado contiene una descripción del caso de estudio implementado y los resultados obtenidos de la implementación del algoritmo SPEA. En la sección caso de estudio y resultados se presentan los casos de estudio real e hipotético, así como los resultados de las soluciones obtenidas y su análisis. En la sección de conclusiones se presentan las conclusiones más relevantes obtenidas en el desarrollo del trabajo. Finalmente, en la sección referencias se presentan las referencias que se consultaron para conocer el estado actual del arte y aplicaciones similares a la del presente trabajo.

\section{OPTIMIZACIÓN MULTI OBJETIVO MEDIANTE ALGORITMOS EVOLUTIVOS}

Basado en los procedimientos evolutivos se han desarrollado diferentes técnicas para resolver problemas de optimización con uno o más objetivos. No obstante, muchas de ellas tienen limitantes al tratar de solucionar un problema de esta índole. Como ejemplo, aquellas técnicas que requieren evaluar funciones objetivo y restricciones diferenciables para operar adecuadamente. Adicionalmente, muchas sólo trabajan con una única solución, lo cual dificulta la obtención de un frente de Pareto óptimo. Los algoritmos evolutivos, tienen la propiedad que, dentro de una única iteración evalúan y encuentran un grupo de soluciones factibles al problema de optimización, logrando hallar con relativa facilidad un conjunto de soluciones que satisfacen las condiciones de optimalidad de un problema multi-objetivo y no requieren conocer si las funciones evaluadas son diferenciables o no. Esto permite al algoritmo evolutivo, operar en espacios de solución complejos y no necesariamente convexos para garantizar su operatividad, además de poder trabajar sobre frentes de Pareto con discontinuidades Zitzler y Thiele (1999).

\section{b. Conceptos de Optimalidad de Pareto}

Los problemas de optimización no necesariamente requieren de una sola función objetivo, por lo que el proceso de optimización se convierte en un problema multi-objetivo, por lo tanto debe definirse una estrategia adecuada para evaluar las funciones y poder definir una solución optima o un conjunto de ellas que cumplan con las restricciones del problema. Diferentes estrategias se han empleado para resolver este tipo de situaciones, siendo los más empleados el método de suma ponderada, método de programación por metas, asignación de prioridades y, por último, los métodos basados en el concepto de optimalidad de Pareto, siendo este ultimo el más encontrado en las aplicaciones actuales.

Una de las características que diferencian los problemas multi-objetivo de los mono-objetivo radica en la posibilidad que los primeros posean un conjunto de soluciones que satisface las condiciones y restricciones planteadas para el problema a resolver, pero el conjunto de soluciones que cumplen con las condiciones anteriores, se caracteriza porque generalmente entran en conflicto al tratar de buscar el optimo, considerando que al tratar de minimizar algunas otras son aumentan de valor o viceversa.

Algunos algoritmos Multi-objetivo hacen uso del concepto de optimalidad denominado Edgeworth-Pareto óptimo, el cual es más conocido como Pareto óptimo. El concepto de optimalidad de Pareto define que una solución es un óptimo, siempre que no exista ninguna otra solución que disminuya o mejore alguno de los objetivos sin desmejorar otro simultáneamente Zitzler y Thiele (1999).

El algoritmo multi-objetivo SPEA genera soluciones que se obtienen a través de la siguiente expresión:

$$
S(i)=\left|\left\{j / j \in P_{t}+\bar{P}_{t} \wedge i \succ j\right\}\right|
$$

Donde,

S: es una solución.

$i: \quad$ es cada individuo.

$P_{t}$ : es población.

$\bar{P}_{t}$ : es un archivo que almacena los valores de $i$.

En la Fig. 1 se representan los conceptos de optimalidad de Pareto para el caso de un problema con dos objetivos, permitiendo de esta forma visualizar como son definidos los conjuntos de Pareto optimo y como se identifican las soluciones dominadas y no dominadas para el problema. 


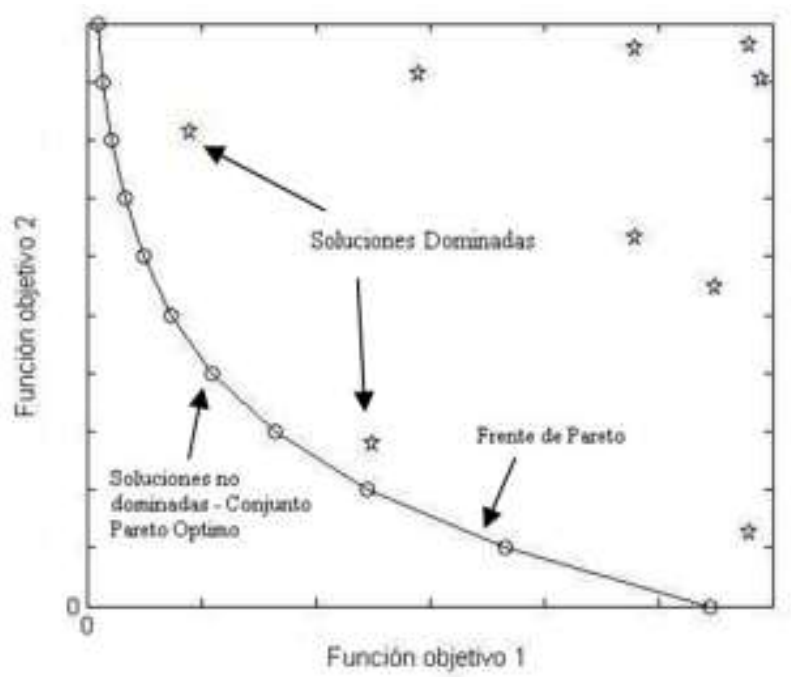

Fig.1: llustración de conceptos de Optimalidad de Pareto.

\section{b. Strength Pareto Evolutionary Algorithm (SPEA)}

El concepto de optimalidad de Pareto ha sido empleado en diferentes tipos de algoritmos multi-objetivo, pero particularmente se ha aplicado en el desarrollo de los algoritmos evolutivos, como el implementado en el presente trabajo. Una revisión detallada de los tipos de algoritmos evolutivos multi-objetivo puede encontrarse en Rivas-Davalos et al. (2007).

Dentro de los algoritmos evolutivos basados en conceptos de optimalidad de Pareto, uno de los más destacados por su buen desempeño en aplicaciones prácticas es el SPEA Coello et al. (2007), el cual fue propuesto por Zitzler y Thiele (1999).

La estructura general de los algoritmos evolutivos multi-objetivo se basa en la misma estructura de los monoobjetivo, empleando los operadores convencionales como son: mutación, cruce y selección. Algunos algoritmos multi-objetivo difieren de los mono-objetivo en que poseen de manera general una población adicional externa, dentro de la cual se alberga el frente de Pareto de la generación en curso, la cual es remplazada según evolucionan los individuos de las poblaciones siguientes.

El algoritmo SPEA se diferencia de otros de su tipo en que: a) posee una población externa con los individuos no dominados, los cuales constituyen el frente de Pareto de la población en curso; b) la asignación de la aptitud de los individuos se efectúa en función de la capacidad de dominio de la población externa sobre los individuos de la población general; c) todas las soluciones participan de la selección, sin excluir ningún individuo y d) se emplea un método de agrupamiento para reducir el tamaño de la población externa, y con él, se garantiza además la diversidad de la población. Para el algoritmo implementado, se ha empleado el método de agrupamiento Fuzzy C-means en Xu y Wunsch (2005). Este método es adecuado porque reúne características similares de varias comunidades 0 individuos y obtiene sus rasgos más eficientes 0 sobresalientes, los cuales pasarán a una siguiente generación.

Parte primordial del algoritmo es la asignación de la aptitud o fitness, la cual se encuentra dividida en dos partes, dependiendo de la población a la cual se le está asignando.

\section{CALIDAD DE LA ENERGÍA EN LA PLANEACIÓN DE LA DISTRIBUCIÓN}

En la actualidad el problema de calidad de la energía en la planeación de los sistemas eléctricos de distribución no solo trata de disminuir el número de interrupciones del sistema o número de clientes afectados por las mismas, sino también intenta disminuir el impacto debido a otrasperturbaciones que ocasionan grandes pérdidas a los consumidores, como son los SAGs (García, 2012; García 2011).

Los SAGs son unos de los problemas más comunes de la calidad de la energía en los sistemas eléctricos. Estos provocan diariamente pérdidas económicas por daños en maquinaria, equipo electrónico y otros equipos eléctricos sensibles a las disminuciones en el voltaje de alimentación. Estoha motivado a las empresas de distribución a trabajar en mejorar la capacidad de respuesta del sistema ante las fallas que ocasionan los SAGs, quienes son finalmente los causantes de las pérdidas García (2012). 
Existen dos métodos ampliamente utilizados para el cálculo de SAGs en sistemas eléctricos, el primero denominado el método de posicionamiento de Falla y el segundo el de distancia crítica Olguín y Bollen (2002). El método de posicionamiento de falla requiere construir la matriz de admitancia del sistema, esto lo hace un método adecuado para sistemas mallados, como los sistemas de transmisión, donde se requiere generar matrices de impedancias de secuencias (positiva, negativa y cero) del sistema para cada tipo de falla y cada lugar donde se requiera establecer la falla. Desde luego se generarán muchas matrices y de grandes dimensiones, lo que implica un gran esfuerzo computacional. Con estas matrices se calculan las corrientes y voltajes de falla en todos los nodos del sistema, aplicando el método de componentes de secuencia.

El método de distancia crítica está basado en el cálculo de divisor de tensión mostrado en la Fig. 2, es de fácil cálculo para sistemas radiales considerando que no requiere de la construcción ni solución de la matriz de admitancias del sistema. Los cálculos operacionales disminuyen dramáticamente debido a que todo el sistema se reduce a un circuito equivalente Thevenin como el de la Fig. 2.

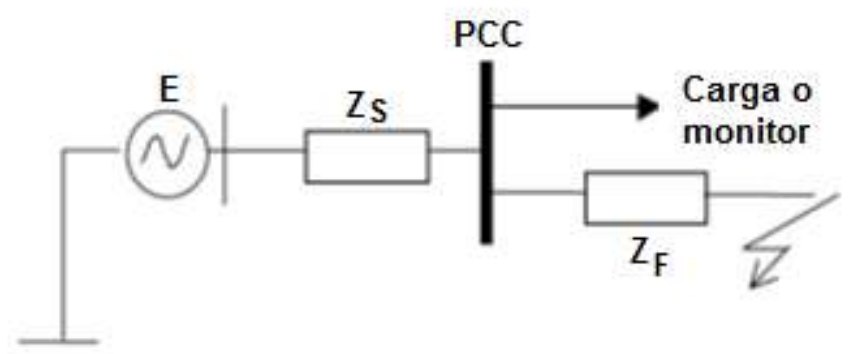

Fig. 2: Divisor de voltaje para el cálculo de la magnitud del hueco.

En la ecuación 2 puede apreciarse la expresión para el cálculo del SAGs, en por unidad, en el Punto de Acoplamiento Común (PCC).

$$
v_{\text {sag }}=\frac{Z_{F}}{Z_{S}+Z_{F}}
$$

Debido al poco peso computacional que requiere el método de distancia crítica para el cálculo de SAGs, éste es empleado en técnicas estocásticas de predicción de SAGs o en aquellos métodos que demanden de gran número de simulaciones, como es el caso del presente artículo. En otros trabajos se ha empleado datos tomados de modelos estocásticos que tratan de predecir el número de SAGS esperados en un punto del sistema Olguín y Bollen (2002), pero este método se hace inviable debido al peso computacional de estos cálculos y que sus resultados no pueden generalizarse para otros entornos ni sistemas de distribución.

\section{FORMULACIÓN DEL MODELO DE PLANEACIÓN}

Para la definición del modelo de planeación propuesto en el presente trabajo, se tienecomo funciones objetivo la necesidad de minimizar las inversiones por expansión en el sistema de distribución, considerando los costos por las perdidas técnicas anuales asociadas a la cargabilidad o la potencia transportada por los conductores y la necesidad de responder a niveles de calidad de la energía más exigentes desde el punto de vista de la regulación, lo que se traduce en la minimización de los SAGs esperados en el sistema durante un año. La obtención del número de SAGs experados (que se presenta en la ecuación (6)) se realiza basado en la generación de fallas a partir de la longitud del tramo donde se presenta, ubicación dentro del tramo, tipo de falla, tipo de construcción, nodo inicial, nodo final, impedancias de secuencia positiva y negativa. La minimización del número de SAGs consiste en seleccionar tipos de construcción adecuados, impedancias de secuencias positivas y negativas que disminuyan el impacto de la falla, todo esto traerá consigo un incremento en el costo de la solución, por lo que hay que encontrar la solución más óptima, lo cual se consigue con la implementación del algoritmo SPEA, el cual se presenta en la sección V.

El modelo adoptado para cumplir con estas necesidades es planteado para un único periodo de un año y se implementó empleando como funciones objetivo las expresiones (5) y (7).

$$
\begin{aligned}
& \mathrm{f}_{\text {costo }}=\sum_{\text {Tramo }=1}^{N}\left(\mathrm{X}_{\mathrm{ij}}{ }^{*} \mathrm{C}_{\mathrm{fk}}{ }^{*} \mathrm{~L}_{\mathrm{ij}}\right) \\
& \mathrm{f}_{\text {cos-vble }}=\sum_{\text {Tramo }=1}^{N}\left[\left(\mathrm{l}_{\mathrm{ij}}+\mathrm{I}_{\mathrm{ji}}\right)\left(\mathrm{X}_{\mathrm{ij}}{ }^{*} \mathrm{C}_{\mathrm{V}}{ }^{*} \mathrm{~L}_{\mathrm{ij}}\right)\right]
\end{aligned}
$$




$$
\begin{aligned}
& \mathrm{f}_{\text {Total }}=\mathrm{f}_{\text {costo }}+\mathrm{f}_{\text {cos-vble }} \\
& \mathrm{C}_{\mathrm{V}}=8760\left(\mathrm{C}_{\mathrm{kWh}}\right) \mathrm{F}_{\mathrm{p}} \mathrm{R}_{\mathrm{ij}} \frac{\mathrm{s}_{\mathrm{ij}}^{2}}{\mathrm{~V}^{2}}
\end{aligned}
$$

Las componentes de las ecuaciones 3-6 son las siguientes:

$f_{\text {costo: }}$ función de costo de construcción

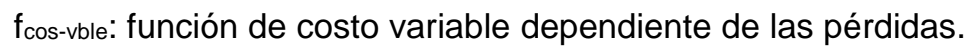

fotal: es la función de costo total o función objetivo a optimizar.

i: $\quad$ es el nodo inicial.

$\mathrm{j}$ : es el nodo final.

$\mathrm{x}_{\mathrm{ij}}$ : es el tramo entre los nodos i-j (Variable binaria)

$\mathrm{L}_{\mathrm{ij}}$ : es la longitud del tramo entre los nodos $\mathrm{i}-\mathrm{j}$.

Cfk: son los costos fijos asociados a la construcción del tipo de red K.

$\mathrm{C}_{\mathrm{V}}$ : son los costos variables debidos a las pérdidas del sistema.

$\mathrm{C}_{k W h}$ :son los costos por Kilovatio-hora.

$\mathrm{F}_{\mathrm{p}}$ : es el factor de pérdidas definido por el operador de red.

$\mathrm{R}_{\mathrm{ij}}$ :es la resistencia del conductor entre los nodos $\mathrm{i}$-j.

$\mathrm{S}_{\mathrm{i} j}$ :es la potencia que circula a través del conductor $\mathrm{i}-\mathrm{j}$.

V:es la tensión nominal del circuito.

lij:es la corriente que circula por el tramo i-j.

$\mathrm{K}$ :es un valor entero que representa un tipo de red.

$\mathrm{N}$ : número de tramos del caso de estudio.

Aunque el número de nodos totales es fijo, en las ecuaciones (3) a (6) ellos varían a medida que se van recorriendo los tramos en el sistema de distribución planteado, todo esto con el fin de obtener un barrido completo del sistema.

El número de SAGs esperado para el circuito se calculó de acuerdo con la ecuación 6 .

$$
\text { SAGs }=\lambda_{3} \sum_{F_{N}=1}^{N} S_{N}+\lambda_{2} \sum_{F_{N}=1}^{N} S_{N}+\lambda_{1} F_{N=1}^{N} S_{N}
$$

Las componentes de la ecuación (7) son las siguientes:

$\mathrm{S}_{\mathrm{N}}$ : número de SAGS registrados ante la falla simulada en el tramo $\mathrm{N}$.

$\lambda_{3}$ : porcentaje de fallas trifásicas al año.

$\lambda_{2}$ : porcentaje de fallas bifásicas al año.

$\lambda_{1}$ : porcentaje de fallas monofásicas al año.

$\mathrm{F}_{\mathrm{N}}$ : Falla simulada en el tramo N.

Vale la pena aclarar que los parámetros $\lambda$ se obtienen mediante el análisis estadístico de los tipos de fallas propias en la región donde se está desarrollando el estudio.

Finalmente para completar el modelo adoptado se definieron las correspondientes restricciones técnicas que garantizaran la factibilidad de las soluciones evaluadas durante el proceso de optimización.

A continuación se citan las restricciones definidas para el proceso en mención:

Límites de capacidad de potencia en conductores: la cual es determinada por las características de los conductores empleados.

Estructura radial del sistema de distribución: esta restricción es verificada contabilizando el número de nodos y el número de tramos que componen el sistema, validando la siguiente condición: número de tramos = número de nodos - 1 .

Límites de tensión según la legislación actual: tomando como referencia valores entre 0.9 y 1.1 como voltajes mínimo y máximo, respectivamente.

Ecuaciones de balance de potencia en los nodos: con los cuales se realiza una sumatoria entre la potencia de entrada y las potencias de salida en cada nodo del sistema.

Garantizar la entrega de potencia en todos los nodos proyectados dentro del sistema: se realiza una 
verificación de que la potencia entregada en los nodos que poseen cargas asociadas, es suficiente para atender la demanda del nodo.

Que las soluciones contengan todos los nodos proyectados del problema de expansión evaluado: para que una solución se considere como factible, se verifica que ésta incluye la atención de la demanda futura en todos los nodos proyectados, para garantizar que se atienden las cargas futuras completamente.

\section{ALGORITMO IMPLEMENTADO}

Como se expuso al inicio, en el presente trabajo se emplea un algoritmo evolutivo tipo SPEA (Strength Pareto Evolutionary Algorithm). Adicionalmente se requirió de la construcción de dos algoritmos complementarios: uno para la validación de restricciones y otro para la cuantificación de los SAGS esperados. A continuación se presenta el detalle de las tres implementaciones realizadas. La salida del algoritmo de restricciones y los insumos del algoritmo para obtención del número de SAGs sirven como insumo o dato de entrada al primer algoritmo (SPEA), por lo que es posible relacionar la salida del algoritmo SPEA (solución) con el número de SAGs generados en cada solución.

\section{a. Implementación del Algoritmo Evolutivo (SPEA)}

En la fase inicial del algoritmo se introducenlos datos del caso de estudio que será procesado. Éste debe incluir el detalle de la identificación de las posibles rutas que son propuestas como viables, la configuración actual de la red, la proyección de demanda por nodo en el periodo de estudio, tanto para los nodos existentes como para los proyectados, que se obtiene de un estudio de demanda de energía particular para cada región, ciudad o país, el cual en el presente trabajo es tomado como insumo suministrado por un estudio previo de otra naturaleza. La identificación de las rutas viables que se introducen al problema se realiza mediante una evaluación de la topología del terreno considerando obstáculos naturales, edificaciones, acceso a los terrenos y, naturalmente, considerando la experiencia del planeador. Además se requiere de algunos detalles adicionales que serán tratados en la siguiente sección.

Una vez introducidos los parámetros iniciales se procede a introducir la población inicial, la cual ha sido definida previamente, teniendo cuidado de haber incluido individuos factibles para entregar un buen punto de partida para el algoritmo.Simultáneamente con la carga de la población inicial se crea la población externa que albergará el frente de Pareto hallado dentro del proceso de optimización. Laspoblaciones están constituidos por $\mathrm{N}$ individuos.

Dado que la codificación empleada dentro del modelo es entera, cada uno de los genes que componen los individuos solo puede asumir valores numéricos otipos de construcción que sean considerados dentro del caso. Aquellos genes que tengan asignado como valor 0 , equivalen a tramos que no son considerados dentro de la solución propuesta.

Una vez definidos los parámetros del algoritmo, población inicial y población externa, se evalúa el dominio de los individuos que componen la población general, agregando los individuos nodominados a la población externa, la cual contendrá el frente de Pareto resultante del proceso de optimización. Si el número de individuos que posee la población externa excede el valor de N (Número máximo de individuos de la población externa), se ejecuta una reducción de la población externa mediante la técnica de Clusterig Fuzzy C-Means. Luego se calcula el Fitness de ambas poblaciones, acorde con los conceptos mencionados en la sección II anterior.

El paso siguiente es aplicar los operadores genéticos definidos para el algoritmo: selección, cruce y mutación. Finalmente se valida como criterio de convergencia cuando se alcance la generación máxima, la cual se definió dentro de los parámetros del algoritmo, igual como sucedió con el cruce y la mutación. Si se ha llegado al límite generacional se guarda el frente de Pareto obtenido en la última generación. Si esta condición no se ha cumplido se repite el proceso desde el paso donde se agregaron los individuos no dominados a la población externa, eliminando los que son cubiertos por otros individuos de la misma población, entendiendo por cubiertos aquellos que no mejoran ninguna de las funciones evaluadas respecto al individuo con el que se le compare.Luego se continúa con el proceso generacional ya descrito, repitiéndose hasta cumplir con la condición de convergencia. En la Fig.3 se muestra el diagrama de bloques del algoritmo descrito.

Los operadores genéticos del algoritmo SPEA tienen algunas particularidades respecto a otros algoritmos de su tipo. Por ejemplo, la selección en este algoritmo se realiza por torneo, pero los padres candidatos son seleccionados inicialmente de manera aleatoria. Con esto se logra dar mayor aleatoriedad a la selección del algoritmo que es elitista por concepción.

El cruce implementado en algoritmos desarrollados para la planeación de sistemas de distribución puede ser 
el mismo que se emplea en cualquier algoritmo evolutivo convencional, pero debe tenerse presente que un cruce en uno o dos puntos e incluso el cruce por segmentos o fracciones de los individuos padre pueden provocar individuos infactibles, lo cual puede ser o no conveniente según se desee. Al desarrollar el algoritmo se implementó un cruce con características especiales que permiten mejorar las deficiencias que presentan los cruces convencionales, el cual se describe a continuación. Para este caso se implementó un cruce homogéneo, el cual inicialmente traslada al hijo las características comunes entre los padres y luego procede a elegir de uno de ellos los genes faltantes, validando que el gen elegido pertenece al padre de mejores características o valor fitness. Con este procedimiento se logra que los hijos hereden efectivamente las buenas características de los padres y con ello se logre obtener descendientes factibles, cosa que no se logra con otro tipo de cruce.

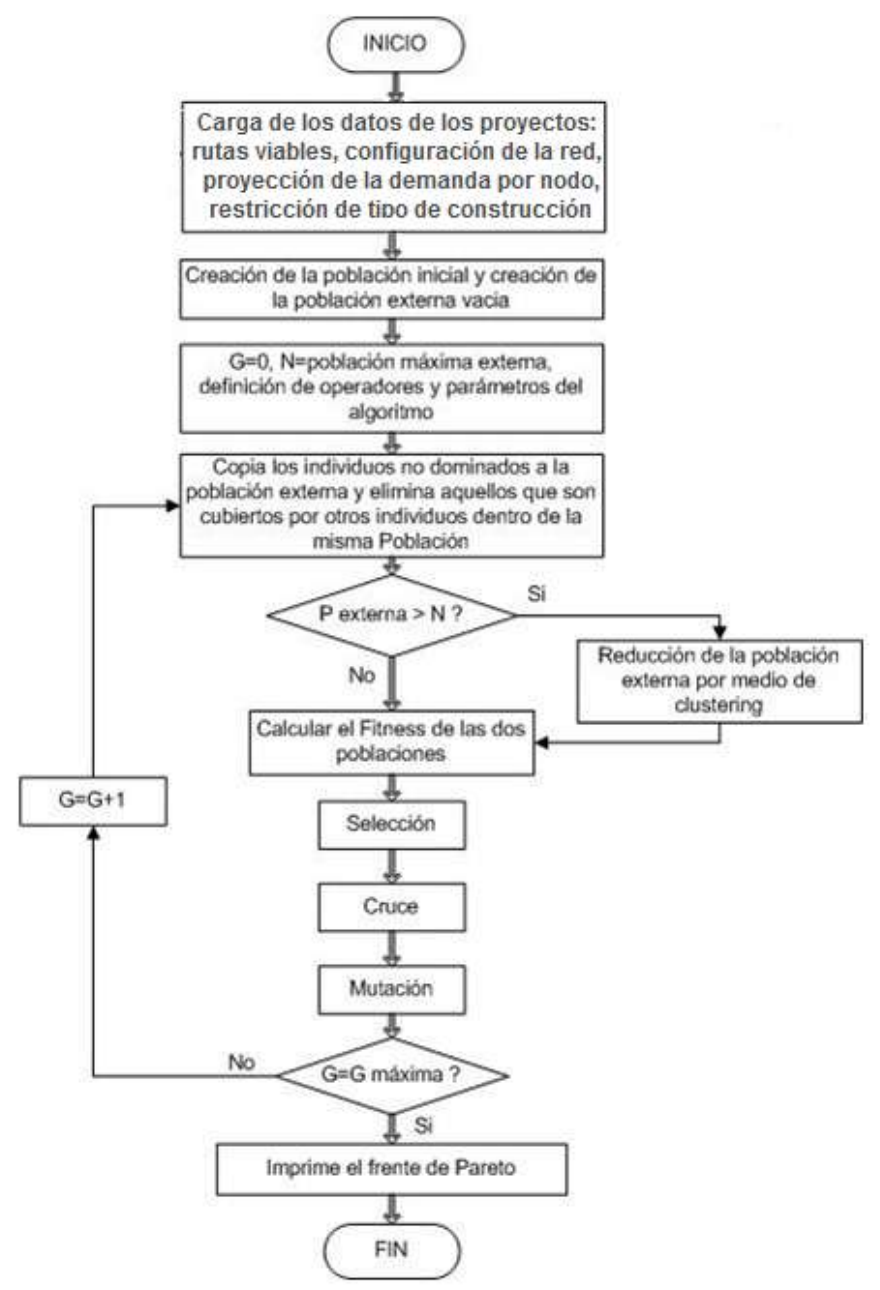

Fig. 3: Algoritmo Evolutivo SPEA.

Finalmente al aplicar el operador mutación, éste se desarrolló de tal modo que solo se realice sobre los tramos proyectados, dado que no tiene sentido realizarlo sobre los ya existentes, además se permitió hacer dos operaciones diferentes: en la primera, si al lanzar la posición aleatoria que mutará se encuentra un tramo con valor diferente de cero, este se mutará por otro tipo de construcción; la segunda es para aquellos tramos que tienen valor diferente de cero, dentro de la cual una vez definido el tramo que mutará se identifica una trayectoria alterna que cumpla con las restricciones radiales del sistema y se cambia la anterior trayectoria por la identificada posteriormente. Con estas estrategias se logra un adecuado equilibrio entre la dispersión en la búsqueda y la infactibilidad constructiva que pueda surgir al aplicar la este operador. Además, la presencia del clustering dentro del algoritmo SPEA permite reducir rápidamente la población externa, eliminando los individuos (o tramos) con peores características y dejando solo los mejores para la siguiente generación. Esto ayuda a reducir la dispersidad de la búsqueda de los mejores individuos, a evitar las no convergencias del algoritmo (o fluctuaciones alrededor de óptimos locales), a lograr más rápidamente la convergencia y en general a obtener tiempos de respuesta mucho menores.

b. Algoritmo Para Validación de Restricciones

Para cumplir efectivamente con el proceso de optimización es necesario efectuar revisiones o validaciones en diferentes etapas del proceso. Dentro de los problemas de planeación de la distribución deben validarse 
dos tipos de restricciones, unas de tipo técnico y otras de tipo constructivo. Las de tipo técnico son validadas mediante flujos de carga radiales (o flujo radial), los cuales entregan el estado de la red en términos de corriente, voltaje y potencia. En la Fig. 4 puede apreciarse el flujo radial implementado para la validación de condiciones técnicas. El flujo de carga radial es el transporte de energía eléctrica en una dirección: desde la fuente de energía hasta las cargas de consumo, sin que haya retroceso o inversión en la dirección de la energía transportada.

Mediante el proceso desarrollado dentro del flujo radial se logra identificar la cargabilidad de los conductores, el perfil de tensiones del circuito correspondiente al caso de estudio, la potencia entregada en cada nodo tanto en proyectados como en existentes y el balance de potencias en todos los nodos que hacen parte del individuo analizado.

Antes de iniciar la simulación del flujo radial se verifican las restricciones de tipo constructivo con lo cual se pretende garantizar la radialidad de la solución, es decir que el algoritmo garantice una sola dirección para el flujo de corriente eléctrica y que esta dirección siempre sea desde la fuente de energía hasta las cargas de consumo, dado que sin esta condición el flujo no puede ser efectuado. De manera complementaria, además de la radialidad se verifica si existen tramos enmallados, es decir aquellos que cierran circuitos y pueden permitir trayectorias de devolución de energía eléctrica, también se verifica si existen tramos que no se hayan considerado dentro de la solución y que comprometan la viabilidad de la misma. Además se verifica que todos los nodos proyectados se encuentren conectados dentro de la solución y que no se presenten tramos sin conectar que sean requeridos para la construcción de la solución.

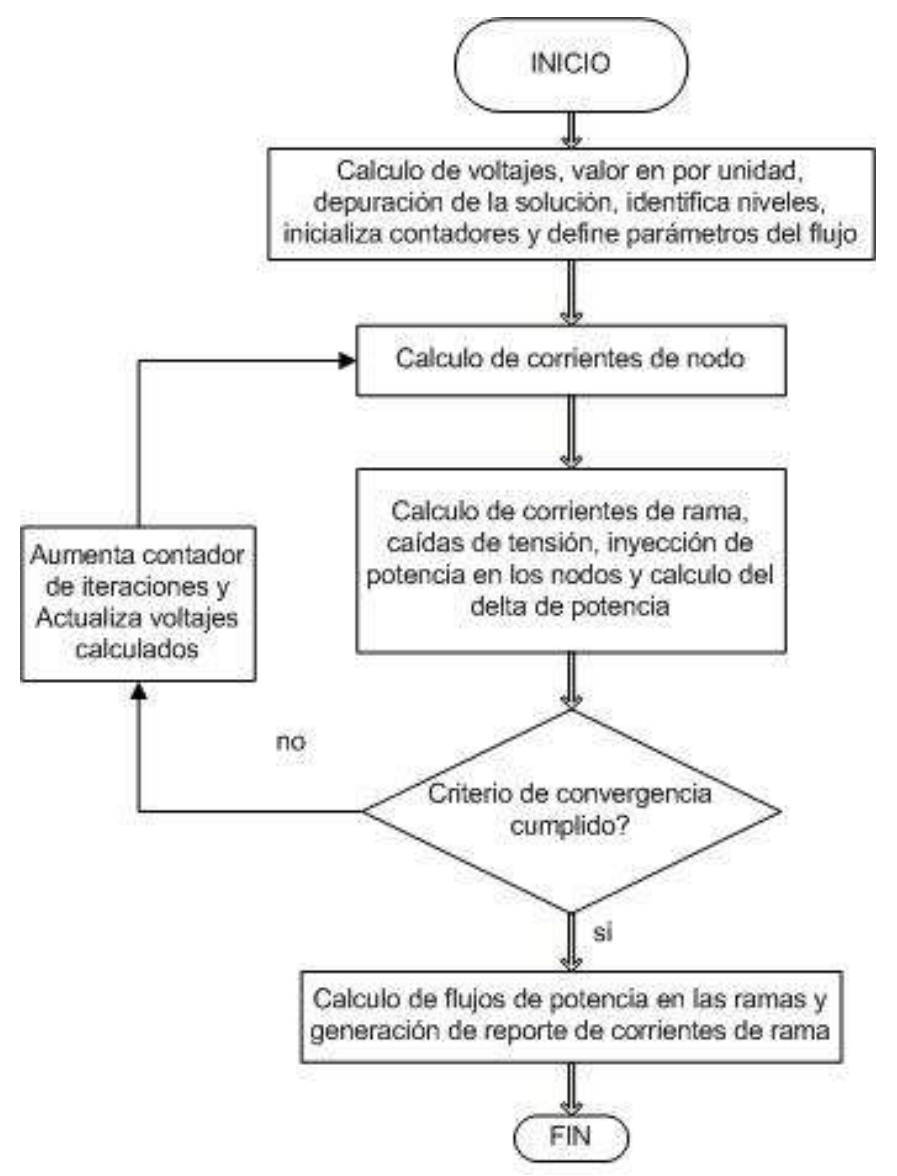

Fig.4: Algoritmo para validación de restricciones.

\section{c. Cuantificación de SAGS esperados}

Una de las funciones objetivos consideradas en este trabajo para la optimización es el número de SAGs esperados por año. La selección de esta perturbación de la calidad de la energía se debe a que afecta dramáticamente a los usuarios por su impacto en los equipos sensibles y la alta frecuencia de aparición. Por ello es necesario empezar a considerarlo en los problemas de expansión de los sistemas de distribución.

Con el objeto de disponer de una forma de cuantificar los SAGs esperados, se implementó un algoritmo que simula las fallas monofásicas, bifásicas y trifásicas que pudieran presentarse en el circuito del caso de estudio. 
El algoritmo fue implementado de modo tal que pueda ser definido el porcentaje de longitud del tramo donde se simulará la falla, esto permite efectuar simulaciones a diferentes distancias de los apoyos, para probar la sensibilidad de la respuesta del sistema según el lugar donde se presenten las fallas.

El algoritmo recibe el individuo que será evaluado y procede a la construcción de una matriz con los datos correspondientes a cada tramo necesarios para la simulación de la falla: longitud, tipo de construcción, nodo inicial, nodo final y las impedancias de secuencia positiva y negativa. Cabe aclarar que esta matriz es de orden operativo y no tiene relación con la matriz de admitancia empleada en el método de localización de falla.

Considerando que es necesario simular los tres tipos de falla mencionadas se implementaron funciones independientes para cada una de ellas, las cuales son integradas luego dentro del algoritmo e invocadas secuencialmente para simular las fallas en su posición correspondiente dentro del tramo.

Las tres funciones que simulan las fallas, retornan al algoritmo el valor de los SAGS registrados en la simulación, el cual es acumulado en memoria para luego cuantificar el total de SAGS esperados aplicando la ecuación 6.

\section{CASOS DE ESTUDIO Y RESULTADOS}

\section{a. Caso de estudio}

Con el fin de mostrar los resultados obtenidos mediante el modelo y algoritmo propuesto se emplearon dos sistemas: uno hipotético de prueba constituido por 24 tramos, entre existentes y proyectados, y el otro real constituido por 73 tramos, incluidos los existentes y proyectados, y 74 nodos. Esto es con el fin de contrastar y verificar los resultados, así como validar el comportamiento del algoritmo SPEA (en cuanto al tiempo de simulación y calidad de las soluciones) en dos situaciones con tamaños y características diferentes.

En la Fig. 5 se presentan la topología del caso hipotético, además de la localización de las cargas que se proyecta ingresar en el horizonte de tiempo definido para el modelo. Aquellos tramos que se encuentran dibujados en líneas discontinuas corresponden a los tramos proyectados, los que se dibujan en líneas sólidas corresponden a los tramos existentes.

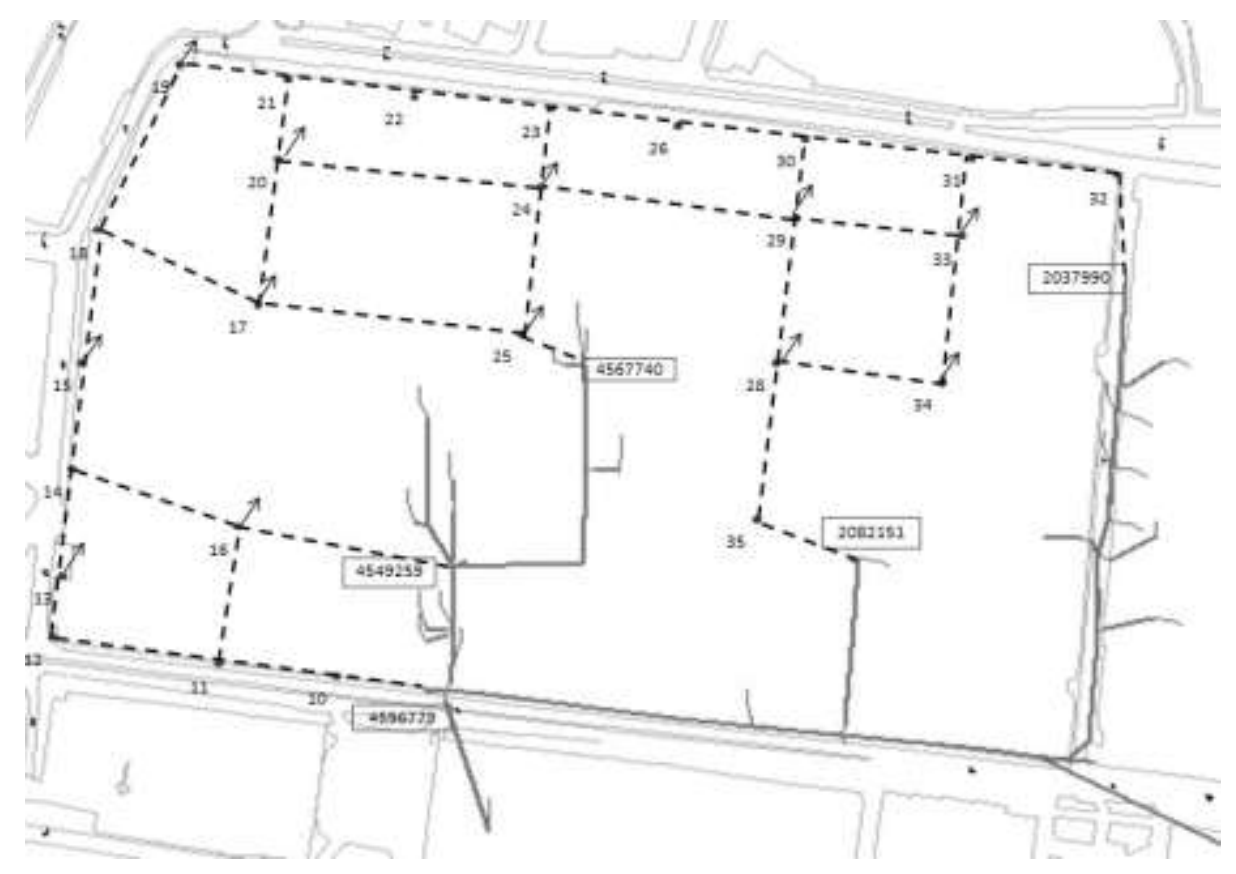

Fig.5: llustración caso de estudio.

Para completar la información requerida para el cálculo del costo de las soluciones y las características eléctricas de cada tramo, en la Tabla 1 se relacionan los costos en USD/m y los valores de las resistencias y reactancias de impedancia negativa y positiva expresadas en $\Omega / \mathrm{km}$. Para poder correr las diferentes soluciones posibles, es necesario conocer el detalle topológico o conectivo del caso. 
Tabla 1: Costos y características por tipo de construcción.

\begin{tabular}{c|c|c|c|c|c}
\hline \hline $\begin{array}{c}\text { Tipo de } \\
\text { construcción }\end{array}$ & $\begin{array}{c}\text { Costo } \\
{[\mathrm{USD} / \mathrm{m}]}\end{array}$ & $\begin{array}{c}\mathrm{R} 1 \\
{[\Omega / \mathrm{km}]}\end{array}$ & $\begin{array}{c}\mathrm{X} 1 \\
{[\Omega / \mathrm{km}]}\end{array}$ & $\begin{array}{c}\mathrm{R} 0 \\
{[\Omega / \mathrm{km}]}\end{array}$ & $\begin{array}{c}\mathrm{X} 0 \\
{[\Omega / \mathrm{km}]}\end{array}$ \\
\hline \hline 1 & 71.22 & 0.58 & 0.30 & 1.15 & 1.19 \\
\hline 2 & 24.08 & 0.58 & 0.45 & 0.93 & 1.34 \\
\hline 3 & 74.72 & 0.24 & 0.26 & 0.81 & 1.16 \\
\hline 4 & 82.50 & 0.24 & 0.26 & 0.60 & 0.95 \\
\hline 5 & 76.89 & 0.29 & 0.42 & 0.64 & 1.31 \\
\hline 6 & 82.50 & 0.29 & 0.42 & 0.52 & 1.20 \\
\hline 7 & 22.34 & 1.48 & 0.48 & 1.89 & 1.64 \\
\hline 8 & 44.53 & 0.37 & 0.17 & 1.16 & 0.54 \\
\hline 9 & 55.67 & 0.37 & 0.17 & 0.98 & 0.44 \\
\hline 10 & 51.22 & 0.42 & 0.16 & 1.21 & 0.40 \\
\hline 11 & 109.72 & 0.14 & 0.14 & 0.47 & 0.13 \\
\hline \hline
\end{tabular}

b. Resultados

b.1 Caso hipotético o de prueba

En la Fig. 6 se observa el resultado de una de las pruebas realizadas al algoritmo con los siguientes parámetros:

Número de tramos:

24;

Tipos de construcción diferentes: 5;

Número de población inicial:

5 individuos;

Limite generacional:

Cruce:

20 generaciones;

Mutación:

1.00

$5 \%$;

En esta figura se destaca la dispersión de las soluciones en el frente de Pareto alcanzado; por tanto, el algoritmo cumple con los fundamentos de optimalidad definidos en la sección II anterior. También muestra que gracias al método de agrupamiento empleado se logra un frente de Pareto compuesto por suficientes individuos y una representación adecuada de ellos gracias a la dispersión mencionada. Bajo estas condiciones el algoritmo alcanzó la convergencia en la generación 20. Finalmente se observa que las soluciones más baratas presentan un mayor número de SAGs esperados por año y viceversa, lo cual es un resultado esperado que confirma la fortaleza del algoritmo.

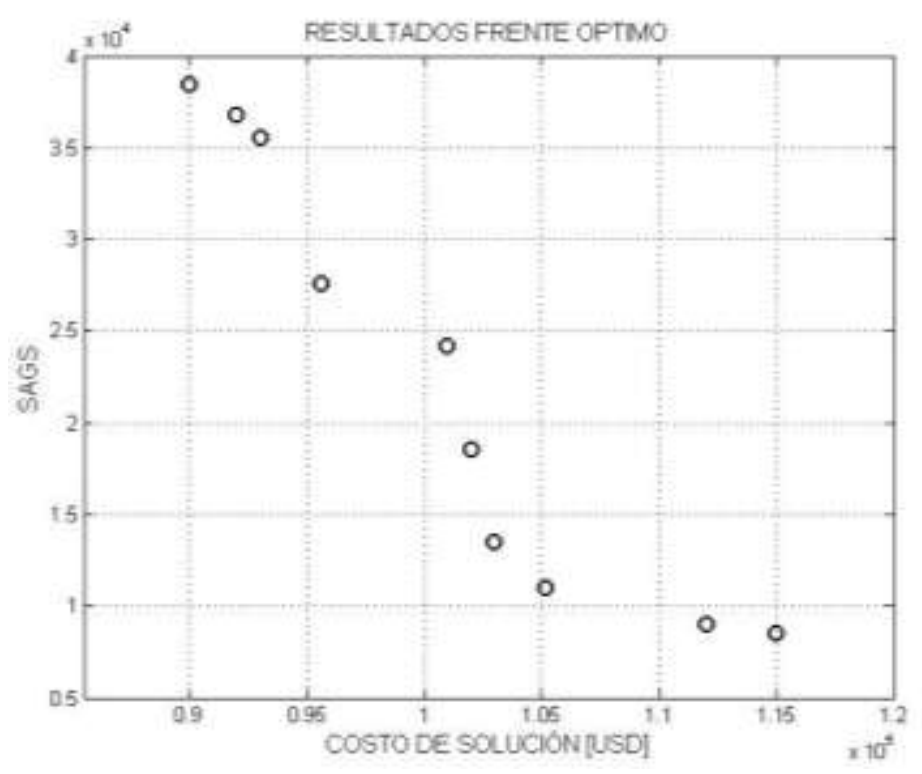

Fig. 6: Ilustración Frente de Pareto Entregado por el Algoritmo. 
Por otra parte, en la Fig. 7 se muestran la evolución del frente de Pareto para las generaciones 1, 9 y 17 obtenidos para un valor fijo de mutación, con la misma población inicial para garantizar siempre el mismo punto de partida y para los mismos parámetros del caso anterior de la Fig. 6, excepto la generación de convergencia (generación 17) y la mutación (2\%).

Algunas pruebas efectuadas mostraron que a medida que aumenta el porcentaje de mutación también aumenta la generación de convergencia, lo cual implica mayores tiempos de simulación, pero mejora notablemente las soluciones óptimas. Por tanto, se deduce que la generación de convergencia no es un parámetro de entrada, sino que es el resultado de la simulación del algoritmo y que depende del porcentaje de mutación establecido y de los demás parámetros de entrada. Estas soluciones al igual que en la Fig. 6 presenta un comportamiento inverso entre el costo de la solución y el número de SAGs esperados por año. Adicionalmente se varió el cruce de 1.00 hasta 1.20, lo cual incremento los tiempos de simulación pero no influyo en el frente de Pareto obtenido.

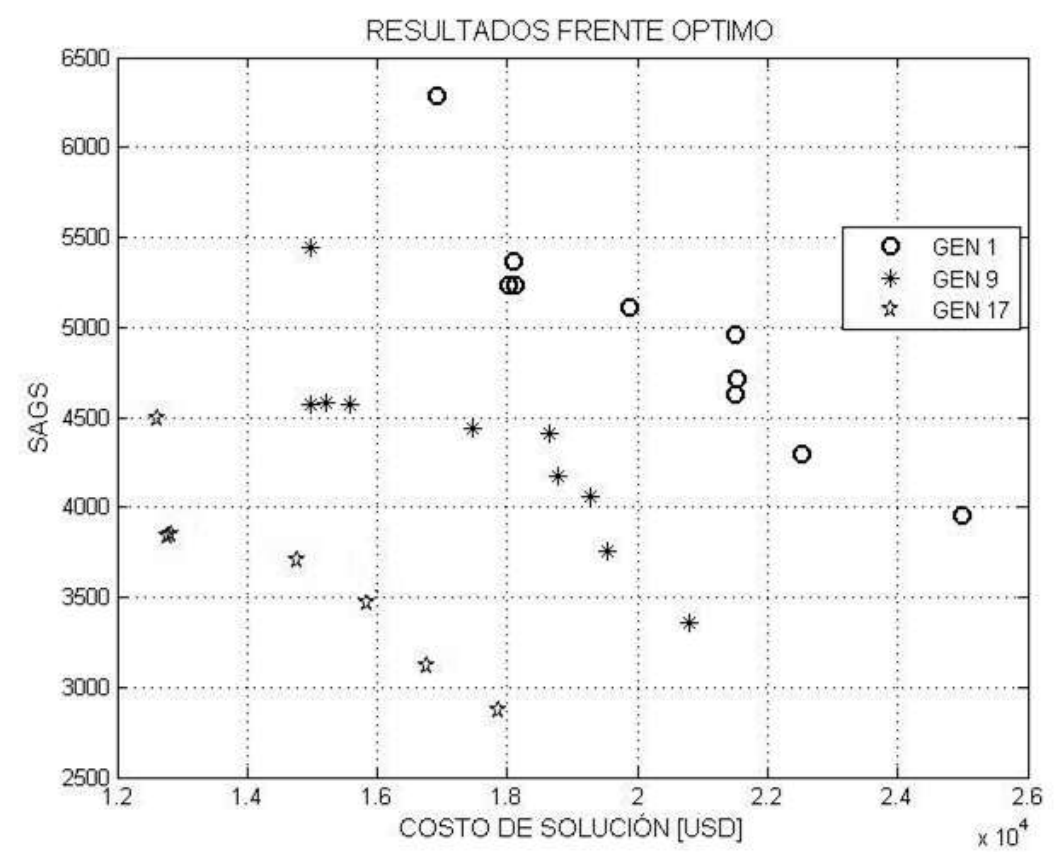

Fig. 7: Ilustración Resultados Caso de 24 Tramos - Convergencia en Generación 17.

\section{b.2 Caso real}

Como se había mencionado antes, este caso cuenta con 73 tramos, incluyendo los proyectados como rutas viables. Las pruebas se realizaron con los siguientes parámetros iniciales:

Número de población inicial: 10 individuos;

Límite generacional: $\quad 30$ generaciones;

Cruce: 1.00 ;

Mutación: $\quad 1 \%, 5 \%$ y $10 \%$;

Partiendo de estos parámetros, en las pruebas se presentó convergencia entre las generaciones 16 y 20 . Al revisar las soluciones se identifica como para las diferentes simulaciones se llega afrentes de Pareto diferentes, pese a partir de la misma población inicial. Otra de las simulaciones realizadas se efectuó variando el cruce de 1.00 hasta 1.20, lo cual incremento los tiempos de simulación pero no influyo en el frente de Pareto obtenido, confirmando de esta manera el comportamiento presentado en el caso hipotético anterior y la validez del algoritmo empleado.

Analizando la Fig. 8 se puede observar que el algoritmo explora diferentes frentes de soluciones hasta llegar a soluciones o valores de inversiones cada vez menores, como el presentado en la generación de convergencia 16 (frente de Pareto). Además, también se nota que los valores de SAGS esperados por año en el circuito disminuyen sensiblemente con el aumento de la generación de convergencia. Las dos condiciones analizadas indican que con una inversión cercana a los USD 100.000 es posible tener en el sistema una expectativa de 550 SAGS por año. Finalmente, al igual que en el caso hipotético se sigue presentando el comportamiento inverso entre el costo de la solución y el número de SAGs esperados por año, confirmando nuevamente la validez del algoritmo. 


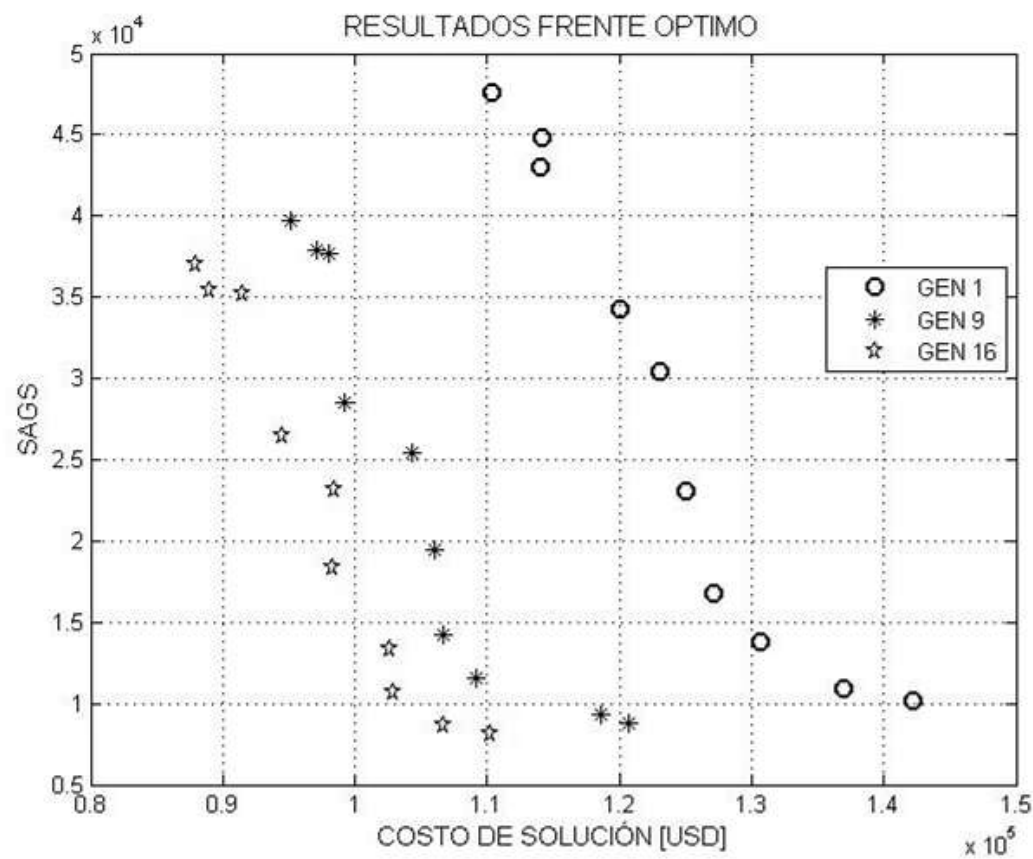

Fig. 8: Ilustración Resultados Caso de 73 Tramos - Convergencia en Generación 16.

\section{CONCLUSIONES}

La metodología de distancia crítica para el cálculo de SAGs es apropiada para ser empleada en planeación de sistemas de distribución debido al bajo peso computacional que esta demanda.

Existe independencia entre los valores obtenidos en las soluciones y el porcentaje de mutación, lo cual confirma la aleatoriedad del algoritmo.

A pesar que el algoritmo es altamente elitista, la exploración de los espacios de solución es adecuada y no cae en óptimos locales.

Al variar los valores del cruce no se presentan beneficios en términos de convergencia y de calidad de las soluciones, en cambio sí representa un incremento considerable en el costo computacional.

El algoritmo multi-objetivo SPEA opera de manera adecuada minimizando simultáneamente los costos de inversión en la expansión y el número de SAGs esperado por año.

La implementación del algoritmo evolutivo multi-objetivo SPEA para la planeación de sistemas de distribución reales es una buena y eficiente herramienta computacional cuando se requiere considerar varias funciones objetivo.

\section{REFERENCIAS}

Carrano, E.G. y otros cuatro autores, Electric distribution network multiobjective design using a problemspecific genetic algorithm, Power Delivery, IEEE Transactions on, 21 (2), 995- 1005 (2006)

Coello, C. A., D. A. V. Veldhuizen y G. B. Lamont, Evolutionary Algorithms for Solving Multi-Objective Problems, Second Edition, MA, Kluwer (2007)

Cossi, A.M. y otros tres autores, Primary power distribution systems planning taking into account reliability, operation and expansion costs, Generation, Transmission \& Distribution, IET, 6 (3), 274-284 (2012)

Diaz-Dorado, E., J.C. Pidre y E.M. Garcia, Planning of large rural low-voltage networks using evolution strategies, Power Systems, IEEE Transactions on, 18 (4), 1594-1600 (2003)

Ganguly, S., N.C. Sahoo y D. Das, Multi-objective planning of electrical distribution systems using particle swarm optimization, Electric Power and Energy Conversion Systems, 2009.EPECS '09.International Conference on, 1-6, 10-12 (2009) 
García, C., E. García y F. Villada, Algoritmo Evolutivo Eficiente Aplicado a la Planeación de la Expansión de Sistemas de Distribución, Información Tecnológica, 23 (4), 3-10 (2012)

García, E., Calidad de la Energía Eléctrica Respecto a Huecos de Tensión, Editorial Académica Española, Berlín (2012)

García, E., New Factor for Voltage Sags Characterization, RevistaFacultad de Ingeniería, 59, 86-97 (2011). Holguin, G. y M. H. J. Bollen, Stochastic prediction of voltage sags: an overview, in Proc. PMAPS Conf. 2, (2002)

García, I., Un enfoque metaheurístico para un problema de ruteo con flexibilidad en las fechas de entrega, Universidad Autonóma de Nuevo León, México (2010)

Lavorato, M. y otros tres autores, A Constructive Heuristic Algorithm for Distribution System Planning, Power Systems, IEEE Transactions on , 25 (3), 1734-1742 (2010)

Lotero, R.C. y J. Contreras, Distribution System Planning With Reliability, Power Delivery, IEEE Transactions on, 26 (4), 2552-2562 (2011)

Mendoza, F. y otros tres autores, Optimal Conductor Size Selection in Radial Power Distribution Systems Using Evolutionary Strategies, Transmission \& Distribution Conference and Exposition: Latin America, 2006, $1-5,15-18(2006)$

Pilo, F., G. Pisano y G.G. Soma, Planning for "high quality" distribution networks, Electrical Power Quality and Utilization, 2007. EPQU 2007.9th International Conference on, 1-6, 9-11 (2007)

Rivas-Davalos, F. y otros tres autores, Evolutionary Multi-Objective Optimization in Power Systems: State-ofthe-Art, Power Tech, 2007 IEEE Lausanne, 2093-2098 (2007)

Xu, R. y D. Wunsch, Survey of clustering algorithms, Neural Networks, IEEE Transactions on, 16 (3), 645-678 (2005)

Zitzler, E. y L. Thiele, Multi objective evolutionary algorithms: a comparative case study and the strength Pareto approach, Evolutionary Computation, IEEE Transactions, 3 (4), 257-271 (1999) 